\title{
Prevalence, Characterization, and Pathogenicity of Salmonella enterica Subspecies enterica Serovar Derby from Yaks in the Aba Tibetan Autonomous Prefecture, China
}

\author{
Xue Fu ${ }^{1,2,+}$, Lan Feng ${ }^{3,+}$, Linghan Kong ${ }^{1,2}$, Chun Li ${ }^{4}$, Xiaodong Zhao ${ }^{5}$, Huade Li ${ }^{6}$, Pengfei Cui ${ }^{1,2}$, \\ Wenjun Yan 1,2, Yaru Zhai 1,2, Lan Zhang ${ }^{1,2}$, Hao Li ${ }^{1,2}$, Hongning Wang ${ }^{1,2}$ and Xin Yang 1,2,*
}

1 Key Laboratory of Bio-Resources and Eco-Environment, Ministry of Education, College of Life Science, Sichuan University, Chengdu 610064, China; 18482195563@163.com (X.F.); m15008448472@163.com (L.K.); cuipengfei@stu.scu.edu.cn (P.C.); yanwenjunibv@163.com (W.Y.); zhaiyr0806@163.com (Y.Z.); zl1007139545@163.com (L.Z.); lihaoibv@163.com (H.L.); whongning@163.com (H.W.)

2 Animal Disease Prevention and Food Safety Key Laboratory of Sichuan Province, Chengdu 610064, China

3 Chengdu Centre for Disease Prevention and Control, Chengdu 610064, China; m18302822161@163.com

4 Sichuan Animal Disease Control Center, Chengdu 610064, China; lich03@163.com

5 Sichuan Longri Breeding Stock Farm, Hongyuan 624400, China; zxd77hzx@126.com

6 Sichuan Academy of Grassland Sciences, Chengdu 610064, China; lihuade78@foxmail.com

* Correspondence: yangxin0822@163.com; Tel.: +86-180-8182-5223

+ These authors contributed equally to this work.

Citation: Fu, X.; Feng, L.; Kong, L.; Li, C.; Zhao, X.; Li, H.; Cui, P.; Yan, W.; Zhai, Y.; Zhang, L.; et al. Prevalence, Characterization, and Pathogenicity of Salmonella enterica Subspecies enterica Serovar Derby from Yaks in the Aba Tibetan Autonomous Prefecture, China. Animals 2021, 11, 2397. https://doi.org/ 10.3390/ani11082397

Academic Editor: Athanasios I. Gelasakis

Received: 24 July 2021

Accepted: 9 August 2021

Published: 13 August 2021

Publisher's Note: MDPI stays neutral with regard to jurisdictional claims in published maps and institutional affiliations.

Copyright: (c) 2021 by the authors. Licensee MDPI, Basel, Switzerland. This article is an open access article distributed under the terms and conditions of the Creative Commons Attribution (CC BY) license (https:/ / creativecommons.org/licenses/by/ $4.0 /)$.
Simple Summary: Salmonella spp. is a very important pathogen in the livestock industry and public health, which poses a major threat to global public health. Yaks and their by-products have a significant economic status in the Qinghai-Tibetan Plateau. The aim of this study was to investigate the prevalence of salmonella in yak farms and to conduct a molecular characterization and tests on its pathogenicity in mice with the use of salmonella isolated from yaks with diarrhea as well as from drinking water samples. The prevalence of salmonella was $19.75 \%$ of 162 samples collected from yak farms, and all isolates were found to belong to the serovar of Salmonella Derby and ST40. All Salmonella Derby isolates from both fecal and drinking water samples from 13 farms were clonally related based on SNP alignment. Salmonella Derby was still detected positively in the feces of model mice on day 24 post-injection. This study reports the prevalence of Salmonella Derby in yaks with diarrhea and in their drinking water. In addition, the pathogenicity of the $S$. Derby in mice was investigated. Findings suggest that Salmonella Derby excreted by diarrheic yaks is a source of contamination for other yaks and the environment and is highly pathogenic to mice. Seeing that Salmonella Derby has become one of the most common Salmonella serovars, this situation gives rise to further risk from the potential spread of food-borne diseases.

Abstract: Salmonella enterica subsp. enterica serovar Derby (S. Derby) is one of the numerous nontyphoidal Salmonella serovars and has been recognized as a food-borne pathogen. In 2019, outbreaks of salmonellosis were reported in 13 yak farms in the Aba Tibetan Autonomous Prefecture, China. A total of 32 salmonella strains were isolated from 162 fecal samples of yaks with diarrhea as well as from drinking water samples. The isolates were subjected to serovar identification, animal experiments, and whole-genome sequencing (WGS) analyses. The serovar of all the isolates was $S$. Derby, and the sequence types (STs) were ST40. The analysis of the differences of single-nucleotide polymorphisms (SNPs) showed that the salmonella strains isolated from 13 farms were clonally related. Animal experiments showed that the lethal dose $\left(\mathrm{LD}_{50}\right)$ was $4.57 \times 10^{7} \mathrm{CFU}$ (colony-forming units); the shedding time of $S$. Derby in mice was 24 days; the bacterial loads in spleen were higher than those in other organs (ileum, liver, and cecum). Pathological analyses by hematoxylin and eosin (H\&E) staining revealed obvious damage in the spleen, liver, and intestine. These results indicate that the $S$. Derby from yaks can cause infection in mice.

Keywords: Salmonella Derby; yak; diarrhea; SNP analysis; virulence 


\section{Introduction}

Salmonella spp. is a very important pathogen in the livestock industry and public health, which poses a major threat to global public health [1]. It is considered to be a multi-host pathogen with long environmental persistence [2]. Since its first isolation, more than 2600 serovars of Salmonella spp. have been confirmed [3]. Except for some serovars that have host specificity such as Salmonella (S.) Gallinarum, most serovars have a wider host range, including chicken [4], pig [5], rabbit [6], sheep [7], and cattle [8], causing considerable economic losses to the livestock industry around the world. Two hundred eighty-seven serovars have been detected in China, which are dominated by $S$. Typhimurium [9]. Salmonella spp. is responsible for $70-80 \%$ of food-borne diseases in China and causes human epidemics, mainly through infection with salmonella-contaminated animals and plants [10].

S. Derby is one of the numerous non-typhoidal Salmonella serovars and has been recognized as a food-borne pathogen [11]. Since its first isolation in 1923, S. Derby has gradually spread worldwide [12-15]. The isolation rate of $S$. Derby in Europe has been increasing year by year; it is also the fourth most frequently isolated serovar in the nonhuman sectors in the United States $[16,17]$. In China, S. Derby has gradually become the main serovar isolated from slaughter pigs [18]. It is prevalent especially in pigs and pork products, with $S$. Derby from pigs being the main source of Salmonella spp. infection in humans [19]. Additionally, in a previous study performed in the United Kingdom between 2014 and 2015, the number of outbreaks increased more than fivefold among turkey flocks, indicating that this serovar is adapted to this particular host [20]. Altogether, S. Derby might represent a significant threat to human health, and the contributions of the pork and poultry sectors deserve to be further investigated.

Yaks (Bos grunniens) are mammals that are adapted to living in regions of high altitude in China, Russia, Nepal, and Mongolia [21,22]. The total yak population is estimated to be 14.2 million, with approximately 95\% in China. Almost 1.52 million yaks live in the Aba Tibetan Autonomous Prefecture, which is located on the southeastern edge of the QinghaiTibetan Plateau. Yaks are essential animals for Tibetans and other nomadic pastoralists in high-altitude environments owing to their provision of the basic necessities (such as milk, meat, transportation, hides for tented accommodation, and dung for fuel) [22], and could thus cause the transmission of salmonellosis to humans through the food products they supply. However, the interspecies transmission of pathogens to yaks has significantly increased due to the complicated environment at high altitudes, where different species of animals share the same ecological resources [23]. Since the first report of salmonellosis in yaks in 1958, salmonella has been isolated and identified in yaks in the plateau area. The number of reports of yak salmonellosis in pastoral areas such as Qinghai, Gansu, Tibet, and Sichuan (Ganzi, Aba) has increased in recent years, suggesting that salmonellosis has become more prevalent among yaks. The Salmonella spp. serovars that infect yak are mainly S. Newport, S. Dublin, S. Saint-Paul, and S. Bovismorbificans, while infection with $S$. Derby has rarely been reported.

The isolation of $S$. Derby from feces of yak diarrhea in a Tibetan prefecture has already been reported in our previous case report in 2017 [24], which had few reports of infection with this serovar. We speculated that it might have been associated with yak diarrhea, but the molecular characteristics and prevalence of this serovar were unclear. In July and August of 2019, an outbreak of salmonellosis in yaks occurred. This study aimed to conduct a comprehensive microbiological investigation of $S$. Derby isolates recovered from clinical infection in yaks, including serovar identification, whole-genome sequencing (WGS) analyses, and its pathogenicity in mice.

\section{Materials and Methods}

\subsection{Sample Collection}

In this study, 13 private yak farms (hereinafter referred to as F1-F13) located in the Aba Tibetan Autonomous Prefecture, with dispersed distribution, were selected; the locations 
of these farms are shown in Figure 1. The size of the farms varied between 300 yaks and 50 yaks; the yaks were free-ranged in their respective areas. We selected yaks with diarrhea symptoms and the number of samples collected in each field is shown in the Supplementary Table S1. The difference in sample sizes was due to the different number of diarrheic yaks at the sampling site. No mortalities were found at the time of sample collection. The average age of animals with diarrhea ranged from one month to three months. The onset of the disease was consistent with the typical clinical signs of diarrhea, i.e., obvious lack of spirit, low appetite, dry nasal mucosa, obvious dysentery. Fecal samples were mostly yellow-green and white; bloody fecal samples mostly watery, occasionally mucous and jelly-like, and accompanied by bad odor.

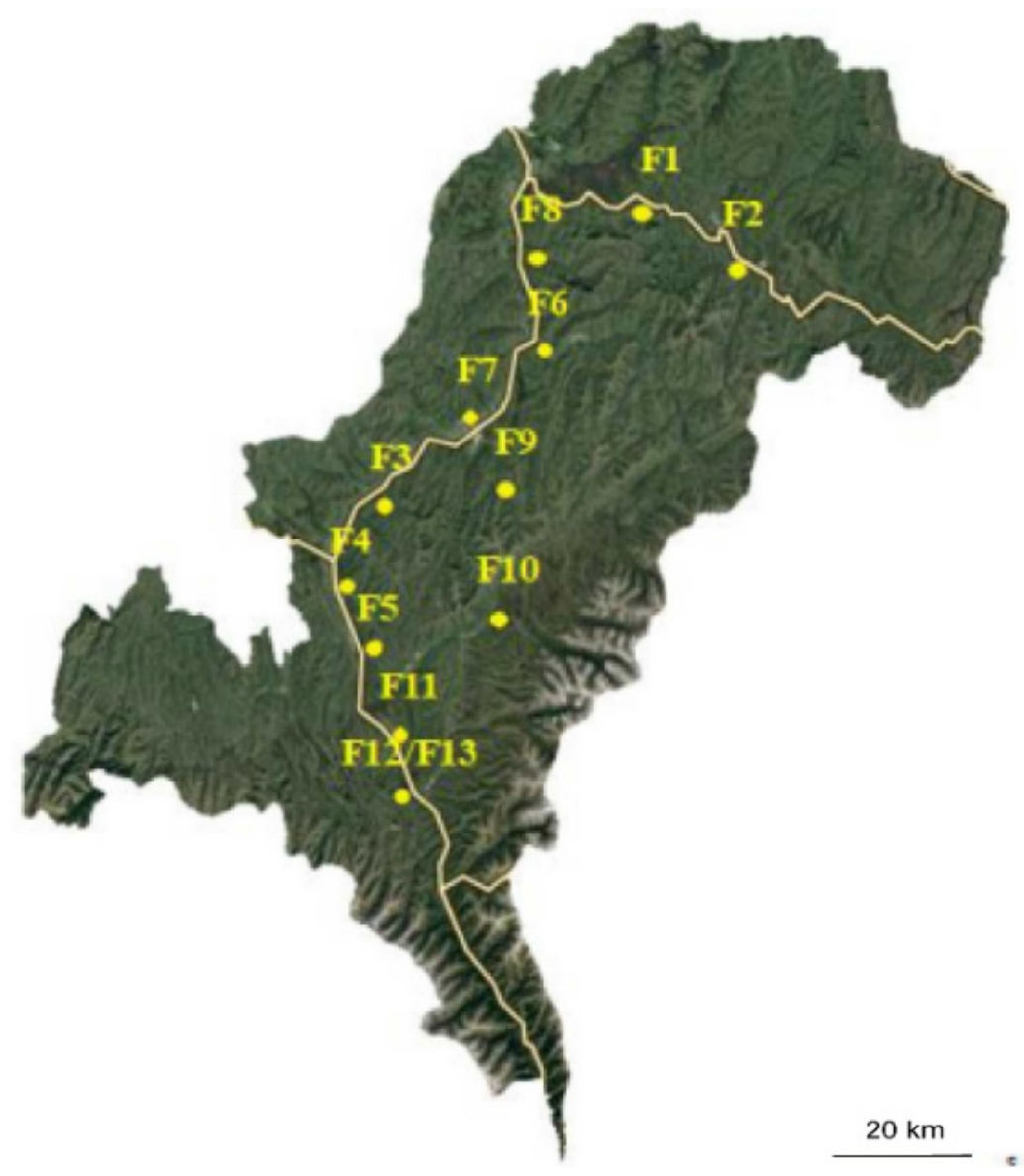

Figure 1. Approximate location of the isolates in the Aba Tibetan Autonomous Prefecture, China. The yellow line represents the river. F1-F13 are abbreviations for Farms 1-13. The latitude and longitude of farms 12 and 13 were the same.

All samples were collected approximately between 5:00 a.m. and 6:00 a.m. (UTC+8) each day when yaks had just been released outdoors and produced fresh feces. The fecal samples were collected using sterilized cotton swabs and placed in aseptic tubes containing $5 \mathrm{~mL}$ of phosphate-buffered saline (PBS, HyClone, Shanghai, China), and drinking-water samples were taken from the river with sterile glass bottles. All samples were packed in sterile plastic bags, transported to the laboratory under cooled conditions, and processed the same day. A total of 162 samples (136 diarrhea fecal samples, 26 drinking water samples) were collected from yaks in 13 farms between July and August in 2019.

\subsection{Salmonella Isolation}

The fecal samples were pre-enriched with buffered peptone water (BPW, Beijing Bridge Technology, Beijing, China, incubated at $37^{\circ} \mathrm{C}$ for $18 \mathrm{~h}$; speed of $180 \mathrm{rpm}$ ), then selected 
and enriched with Rappaport's 119 Broth (MM, Beijing Bridge Technology, Beijing, China, incubated at $42{ }^{\circ} \mathrm{C}$ for $24 \mathrm{~h}$; speed of $180 \mathrm{rpm}$ ), and then isolated on selective xylose lysine tergitol-4 (XLT4) agar (Beijing Bridge Technology, Beijing, China, incubated at $37^{\circ} \mathrm{C}$ for $24 \mathrm{~h}$ ) for salmonella identification. Salmonella detection in water samples was performed with the use of filtration, concentration, pre-enrichment, selective enrichment, isolation by solid selective agar, and detection by the polymerase chain reaction (PCR) method [25]. In short, $500 \mathrm{~mL}$ of water per sample was filtrated using membranes with a pore size of $0.22 \mu \mathrm{m}$ (Beyotime, Shanghai, China). The, membranes were then cut into pieces and placed in $100 \mathrm{~mL}$ of PBS. The solution was transferred into a $50 \mathrm{~mL}$ conical centrifuge tube and centrifuged $(2500 \times g, 30 \mathrm{~min})$. After aspirating the top solution, the remaining concentrated eluate was at $5 \mathrm{~mL}$; the three experimental processes were subsequently conducted. A total of $1 \mathrm{~mL}$ of concentrate was added to $5 \mathrm{~mL} \mathrm{BPW}$ and incubated for $16 \mathrm{~h}$ at $37^{\circ} \mathrm{C}$, with shaking. Then, $1 \mathrm{~mL}$ of the enriched culture was transferred to $10 \mathrm{~mL}$ of $\mathrm{MM}$, after which triplicate parallel experiments were set up. These broths were streaked onto selective XLT4 agar and incubated at $42{ }^{\circ} \mathrm{C}$ for $24 \mathrm{~h}$. According to the Kauffmann-White scheme [26],the isolated salmonella were serotyped using a microtiter agglutination test for $\mathrm{O}$ and $\mathrm{H}$ antigens following the manufacturer's protocols (Diagnostic Sera for Salmonella, TianRun, Ningbo, China). The PCR products were then maintained at $4{ }^{\circ} \mathrm{C}$ and sequenced in both the forward and reverse directions by TsingKe (Chengdu, China). The sequences obtained were analyzed using BLAST [27].

Total DNA of the strain was extracted by boiling a single colony on the plate, as previously conducted [28]. Molecular identification of the isolated strains was achieved by PCR amplification of $16 \mathrm{~s}$ rDNA, with the primers 27F (5'-AGAGTTTGATCMTGGCTCAG-3') and 1492R ( $5^{\prime}$-TACGGYTACCTTGTTACGACTT-3') [29]. Briefly, the cycling program comprised an initial denaturation of $5 \mathrm{~min}$ at $94{ }^{\circ} \mathrm{C}$, followed by 30 cycles of amplification (denaturation at $94{ }^{\circ} \mathrm{C}$ for $1 \mathrm{~min}$, annealing at $50{ }^{\circ} \mathrm{C}$ for $1 \mathrm{~min}$, and extension at $72{ }^{\circ} \mathrm{C}$ for $1 \mathrm{~min}$ ), with a final elongation at $72{ }^{\circ} \mathrm{C}$ for $10 \mathrm{~min}$.

\subsection{Whole Genome Sequencing (WGS) and Bioinformatics Analyses}

A single colony of each isolate was inoculated into $3 \mathrm{~mL}$ of lysogeny broth (LB, Beijing Bridge Technology, Beijing, China) and incubated overnight at $37^{\circ} \mathrm{C}$, with shaking at $250 \mathrm{rpm}$. The samples were stored at $-20^{\circ} \mathrm{C}$ in a brain heart infusion broth (BHI, Beijing Bridge Technology, Beijing, China) containing 20\% glycerol, for later use.

Then, genomic DNA was extracted using a TIANamp Bacteria DNA Kit (Tiangen, Beijing, China) following the manufacturer's instructions. All salmonella isolates were sequenced by Beijing NovoGene Company using the Illumina Nova-seq PE150 technology sequencing platform (Illumina, San Diego, CA, USA) with the paired-end method. The data volume of each library was $\geq 90 \%$ of the target data volume to ensure that the total data quality met the required standard. Raw sequences were assembled through the Enterobase and finally submitted to the GenBank database. The Bioproject numbers of the 32 strains of salmonella in GenBank were PRJNA610941 and PRJNA640879.

Genome annotation was performed by RAST [30]. Multilocus sequence typing (MLST) based on seven housekeeping genes was performed, as previously described [31]. Alleles and sequence types were assigned according to the MLST scheme available online to determine the sequence type [32]. There were core genome MLST (cgMLST) based on 3002 loci and ribosomal MLST (rMLST) based on 53 genes encoding the bacterial ribosome protein subunits $[33,34]$. Single-nucleotide polymorphisms (SNPs) were determined using the CSI Phylogeny tool with its default settings [35]. Then, the evolutionary history was inferred by using the Maximum Likelihood method based on the Tamura-Nei model, and evolutionary analyses were subsequently conducted in MEGA7 [36,37]. An SNP analysis was performed with SD-13-18 (NCBI BioSample: SAMN15339256) as the reference genome, which was isolated in this study. Initial tree for the heuristic search was obtained automatically by applying the Neighbor-Join and BioNJ algorithms to a matrix of pairwise distances estimated using the Maximum Composite Likelihood (MCL) approach, and then selecting 
the topology with a superior log likelihood value. The Newick file was visualized using an online tool, the Interactive Tree Of Life (iTOL) v6 [38]. Five SNPs among isolates were considered to be clonally related and likely to have an epidemiological link $[39,40]$. The web server ResFinder version 4.1 was used for detecting acquired antimicrobial resistance genes [41]. The virulence of isolates was assessed through the detection of Salmonella Pathogenicity Islands (SPIs), while the virulence factors (VFs) were identified by using the SPI database, at the Center for Genomic Epidemiology (CGE) and with the virulence factor database (VFDB), respectively [42,43].

\subsection{Animal Experiments}

Thirty-six 4-6-week-old SPF female Kun Ming (KM) mice (weight 18-20 g) were purchased from the Animal Experimental Center of Sichuan University, China. All mice were housed in plastic cages and kept in an isolated biohazard cabinet for approximately 1 week of acclimatization. All mice had free access to the basal pellet diet and sterilized distilled water, and the cage bedding was changed twice weekly. The mice were treated based on the guidelines of the Committee for the Control and Supervision of Animal Experiments, and all protocols were approved by the Institutional Animal Ethics Committee (License: SYXK (Chuan) 2013-185) of Sichuan University.

The optical density at $600 \mathrm{~nm}\left(\mathrm{OD}_{600}\right)$ of the strain cultures was measured to identify the exponential growth phase and calculate the volume required to administer the correct dose of the strain by the corresponding linear regression analysis. All mice were randomly segregated into six groups (G1-G6; $n=6$ mice per group). The inoculated salmonella strains were centrifuged, washed twice, and resuspended with sterile normal saline (Beyotime, Shanghai, China) to achieve an optical density (OD) value of 0.5 . The mice in G1-G5 were intraperitoneally injected with the following doses of salmonella: $5 \times 10^{5}, 5 \times 10^{6}, 5 \times 10^{7}$, $5 \times 10^{8}$, and $5 \times 10^{9} \mathrm{CFU}$ (colony-forming units); the mice in the control group (G6) were injected with the same volume of sterile normal saline. The number of dead mice for each dose was recorded every day for 30 days, and then the lethal dose $\left(L_{50}\right)$ at different time courses was determined using the Reed-Muench method [44].

From day 3 post-infection (pi) until the end of experiment at day $30 \mathrm{pi}$, feces were collected every 3 days to determine the duration of shedding and the bacterial loads of salmonella following inoculation. The enumeration analyses were performed, as previously described [45]. Briefly, fecal samples were diluted 1:10 in PBS, and serial dilution was performed in the LB until a dilution of $10^{-5}$ was obtained. Then, $100 \mu \mathrm{L}$ of each of the dilutions was placed on three separate XLT4 plates. After incubation at $37^{\circ} \mathrm{C}$ for $24 \mathrm{~h}$, typical black colonies of salmonella were counted to enumerate salmonella in terms of $\mathrm{CFU} / \mathrm{g}$ for each sample.

The spleen, liver, cecum, and ileum from euthanized mice in different groups were removed aseptically and homogenized in PBS to analyze $S$. Derby colonization. The bacterial loads were determined, as described above.

Hematoxylin and eosin (H\&E) staining of the organs (intestine, liver, and spleen) were performed for histopathological examinations. Briefly, after collection during the necropsy, the samples were immediately fixed in $10 \%$ neutral buffered formalin, dehydrated in alcohol, embedded in paraffin, and then stained with H\&E before being observed under a standard light microscope. The images of sections were obtained using a Ba200 digital micro camera (Motic China Group, Xiamen, China).

\subsection{Statistical Analyses}

Data were expressed as mean \pm standard deviation and analyzed using GraphPad Prism Version 8.0.1 (GraphPad software, Inc., San Diego, CA, USA). The normality of the data (i.e., pairwise SNP differences) was checked using the Shapiro test, with R from the pairwise matrix generated by the CSI Phylogeny tool described above [46]. For all tests, $p<0.05$ was used to indicate significant differences between the groups. Organ pathology was evaluated using a histopathological scoring scheme, as previously described [47]. 


\section{Results}

\subsection{Prevalence and Serotyping of Salmonella}

In this study, 32 salmonella isolates were obtained from 162 samples, with $28(20.59 \%$, $28 / 136)$ from fecal samples, $4(17.40 \%, 4 / 26)$ from drinking water samples. Isolates were named using the format SD-F-N (S: salmonella; D: Derby; F: farm number; N: sample number; for instance, the twelfth sample collected from Farm 12 was named SD-12-12). The prevalence of $32 \mathrm{~S}$. Derby strains is as follows: F1(1) (F1(1) indicates that there was one $S$. Derby isolated in farm 1), F2(2), F3(1), F6(2), F8(3), F9(1), F11(1), F12(7), and F13(12). The serovar of all isolates was $S$. Derby.

\subsection{Molecular Typing of S. Derby and Whole Genome Sequencing (WGS) Anaylses}

All 32 S. Derby strains belonged to ST40 and rST28544, and further divided into 3 different cgSTs, mainly cgST198192. Only two strains were cgST198267 (SD-12-10) and cgST198266 (SD-13-17). The distances between each strain was 0-2 in the term of cgSTs. The phylogenetic tree based on the SNPs is shown in Figure 2; a total of 27 SNPs were identified. The SNPs among strains shown in Figure 2 as grouped in line 1, line 2, and line 3 were 0 , indicating that strains from distinct farms were clonally related. Pairwise SNP distance between some strains from different farms were within 5 (shown in Figure 2). Whole genomic analysis of $S$. Derby carried multiple virulence genes, including genes encoding Type III secretion system (T3SS) effectors, adherence (shown in the Supplementary Table S2). In addition, other bioinformation analyses are shown in Supplementary Table S2.
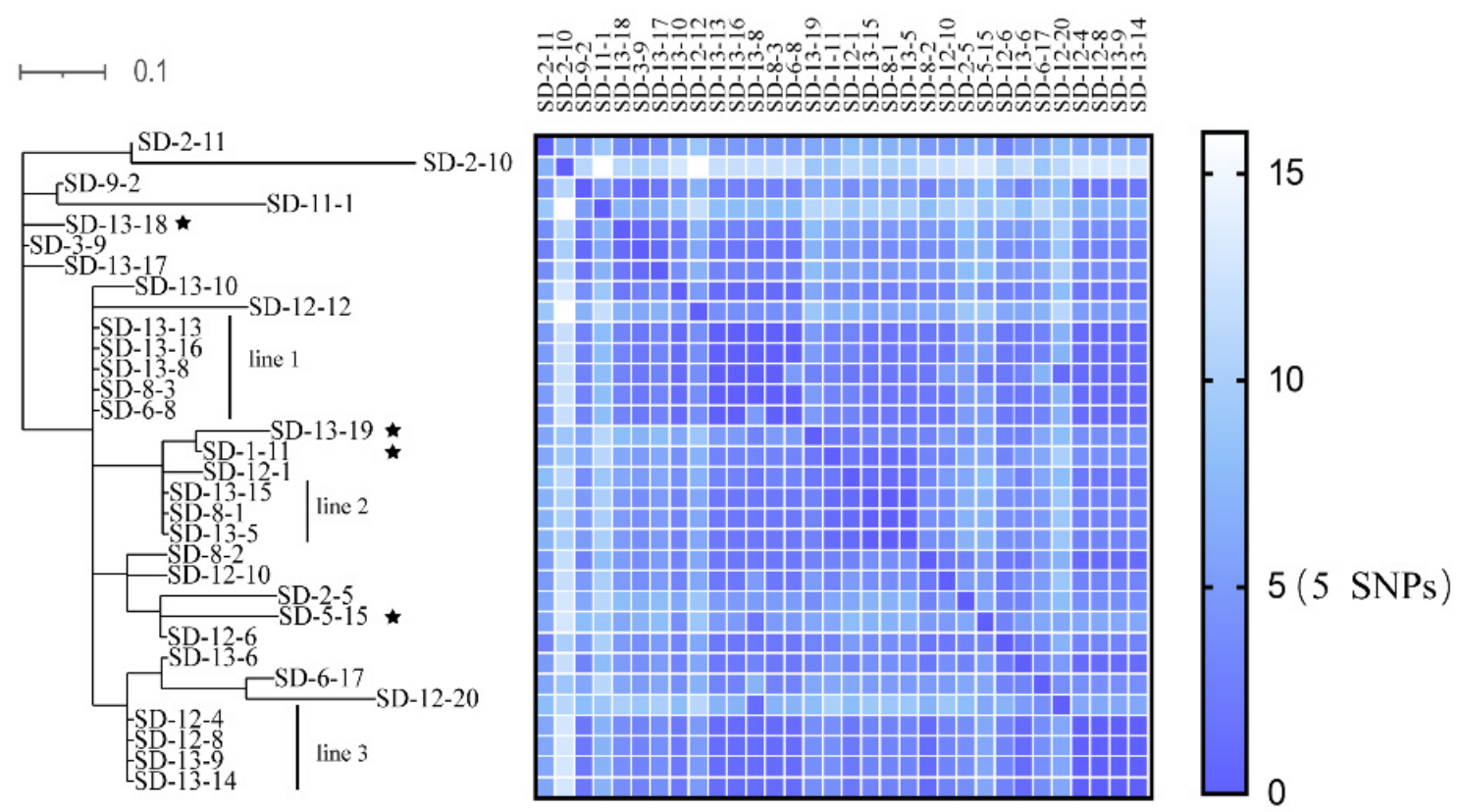

Figure 2. Molecular Phylogenetic analysis by Maximum Likelihood method. Phylogenies were inferred from Singlenucleotide polymorphisms (SNPs) and pairwise SNP distances (0-16 SNPs) are shown using a heat map. The tree is drawn to scale (0.1), with branch lengths measured by the number of substitutions per site. The analysis involved 32 nucleotide sequences. All positions containing gaps and missing data were eliminated. There was a total of 27 positions in the final dataset. SNPs between strains in different lines (line 1-3) were 0. Colors in the heat maps indicate the numbers of pairwise SNP distances between isolates, with blue being the lowest and white being the largest. The star markers indicate that the samples were isolated from drinking water.

\subsection{Virulence of S. Derby in Mice \\ 3.3.1. $\mathrm{LD}_{50}$ Calculation}

The survival curves of the mice challenged with $S$. Derby are shown in Figure 3. All mice in groups G1-G4 $\left(5 \times 10^{5}-5 \times 10^{8} \mathrm{CFU}\right)$ showed diarrheal symptoms, which 
gradually decreased in the experimental process; mice in the $5 \times 10^{9}$ group were dead on day 1 pi. The $\mathrm{LD}_{50}$ was measured as $4.57 \times 10^{7} \mathrm{CFU}$.

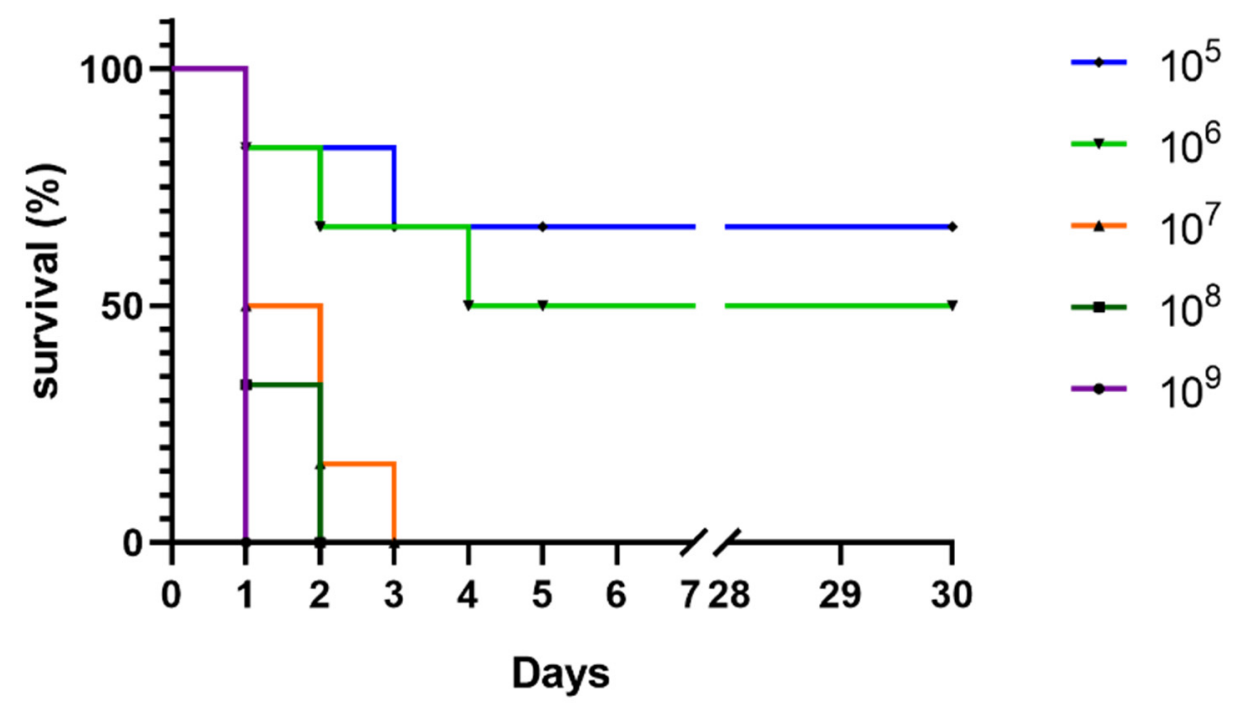

Figure 3. Female Kun Ming (KM) mice were challenged intraperitoneally with $5 \times 10^{5}-5 \times 10^{9} \mathrm{CFU}$ (colony-forming units) of bacteria. Mice survival 30 days after infection is shown; the mortality rate of the strain used was at $50 \%$ during the experimental period.

\subsubsection{Fecal Shedding and Bacterial Loads in Organs}

Fecal shedding was monitored for 30 days. In $S$. Derby-infected mice, salmonella was detected from day 3 pi to day $24 \mathrm{pi}$, ranging from $5.8 \times 10^{4}$ to $4.48 \times 10^{5} \mathrm{CFU} / \mathrm{g}$ of feces (Figure 4). Feces of all animals in the control group remained culture-negative during the entire experiment.

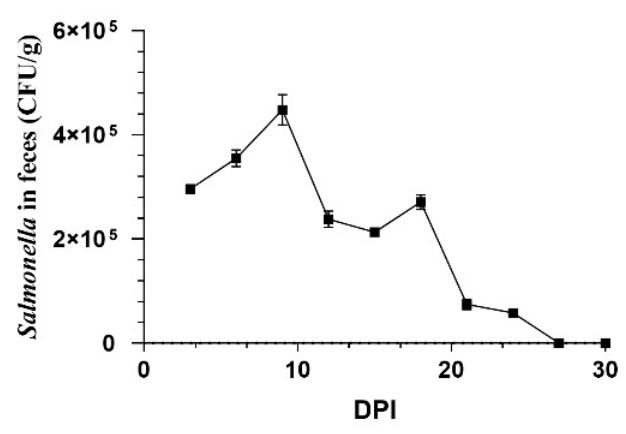

(a)

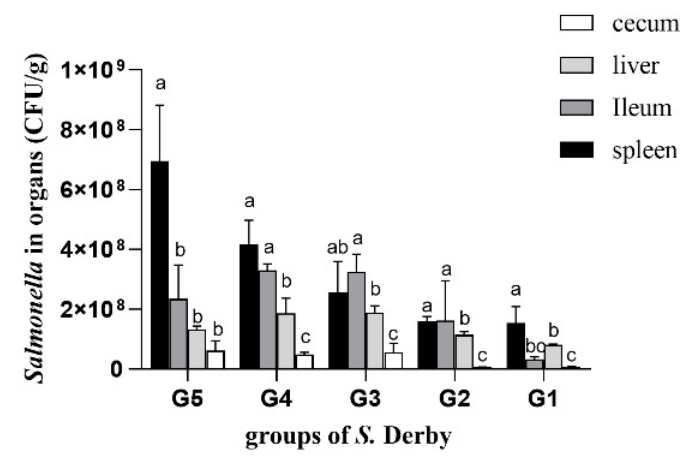

(b)

Figure 4. Fecal shedding and organ bacterial loads of the Salmonella (S.) Derby strain in the mouse model. (a) Fecal pellets were collected from the KM mice at the indicated time points for 30 days (or until the animal was sacrificed). The shedding of $S$. Derby was at about 24 days. Dots represent mean \pm SD (Standard deviation) CFU (colony-forming units) counts in pellets of each mouse group; (b) Colonization in systemic and intestinal sites. Bacterial load in each mouse is represented as CFU/g by the individual column in the spleen (black columns), liver (light gray columns), ileum (gray columns), and cecum (white columns) at different doses. Group1-Group5 (G1-G5): The mice in G1-G5 were intraperitoneally injected with the following doses of salmonella: $5 \times 10^{5}, 5 \times 10^{6}, 5 \times 10^{7}, 5 \times 10^{8}$, and $5 \times 10^{9} \mathrm{CFU}$. Letters indicate significant differences between organs in each group after multiple comparisons.

The results of salmonella colonization in each organ of the mice were ultimately concentrated at $10^{7}-10^{8} \mathrm{CFU} / \mathrm{g}$. Organs of all animals in the control group were culturenegative at day 30 pi. The colonization of $S$. Derby in four organs (spleen, ileum, liver, and 
cecum) had significant difference in all groups ( $p<0.05$; shown in Figure 5). S. Derby loads in the spleen were obviously higher than salmonella colonization in the cecum, followed by the liver in G5-G1. The cecum had the lowest concentration.
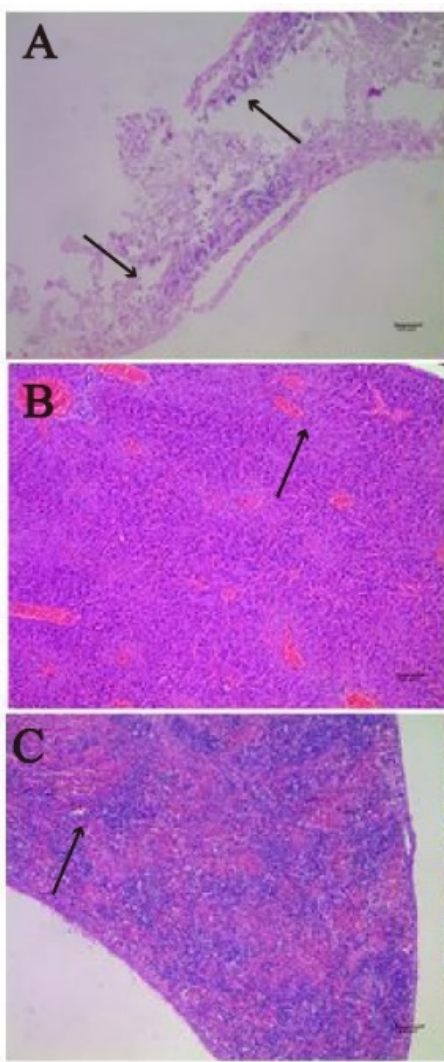
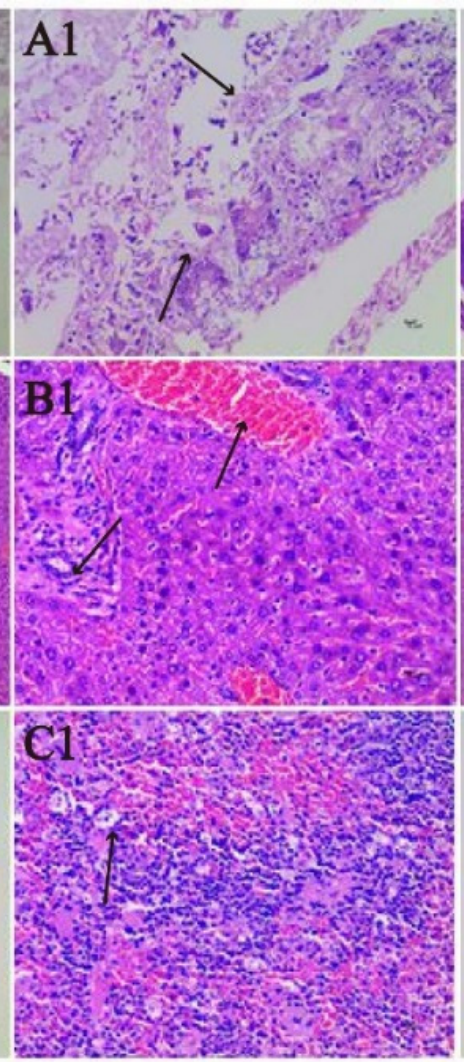
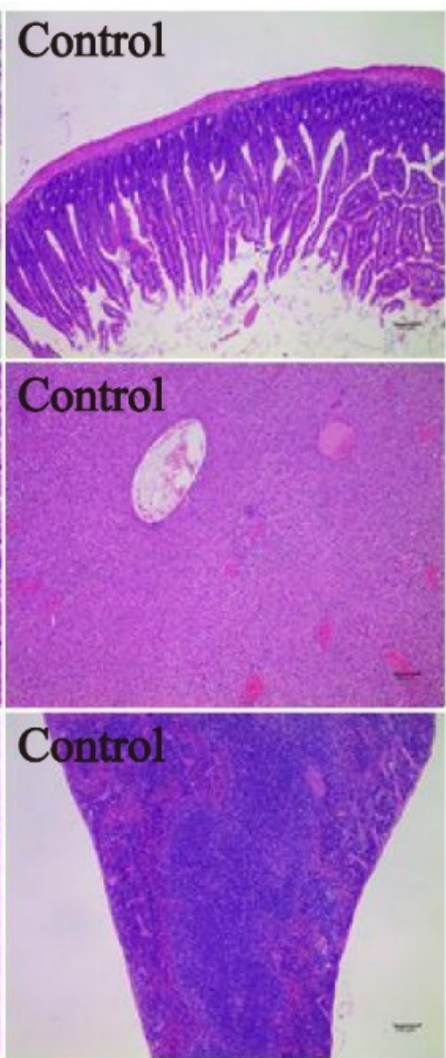

Figure 5. Histological lesions in the spleen, liver, and intestines of KM mice. (A) $(100 \times$ intestine)/(A1) $(400 \times$ intestine): the structure of intestines was destroyed, pointed to by the arrows in (A)/(A1); (B) $(100 \times$ liver $) /($ B1 $)(400 \times$ liver $)$ : heterogeneous cytoplasmic staining results in the empty reticular structure, pointed to by the arrows in $($ B $) /($ B1 $) ;(C) ~(100 \times$ spleen $) /($ C1) $(400 \times$ spleen $):$ nucleolysis was observed, pointed to by the arrows in $(\mathbf{C}) /(\mathbf{C} 1)$. Scale bar: $100 \mu \mathrm{m}((\mathbf{A}-\mathbf{C})$ and the Control group); $10 \mu \mathrm{m}$ (A1-C1).

\subsubsection{Histopathological Analyzation}

The H\&E-stained sections of the spleen, liver, and intestines were subjected to histopathological analyzation. As shown in Figure 5A,A1, S. Derby caused intestinal damage. Severe focal necrosis was observed in the intestinal mucosa, with cell breakdown and disintegration, and some inflammatory cell infiltration (mainly lymphocytes) in the necrotic area. Intestinal gland atrophy was also observed to some extent.

In Figure 5B,B1, liver damage was observed, consisting of vacuolar degeneration of some hepatocytes around the central vein and portal canal area. The cytoplasm of hepatocytes was unevenly reticulated and dissolved to form vacuoles of different sizes. A few inflammatory cells, mainly lymphocytes and a few neutrophils, were scattered in the sinusoids of the liver. The pathological changes in the spleen (shown in Figure 5C,C1) included some lymphocyte infiltration of the white pulp splenic corpuscle, with punctate necrosis and a sparse arrangement of cells. The nuclei of necrotic cells were lost, and the red pulp of the spleen was slightly congested with increased erythrocyte infiltration.

\section{Discussion}

Salmonella spp. is increasingly present in cattle around the world, in those bred for both meat and milk production [48-51]. Guizelini et al. and Salaheen et al. found that most serovars of Salmonella spp. can infect and cause disease in cattle, leading to direct economic 
losses in the dairy industry associated with mortality or body weight loss, in addition to indirect losses caused by reduced feed conversion or medical care costs [52-54]. More than $90 \%$ of yaks in the world live in China and provide local residents with meat, dairy products, transportation, and fuel, which are vital to the meat and dairy production system in high-altitude regions [55]. S. Derby is one of the numerous non-typhoidal Salmonella serovars well-adapted to pig and turkey, and is also pathogenic to humans [56-58]. Since information about the $S$. Derby serovar isolated from yaks is still scarce, the aim of this study was to clinically investigate an outbreak of salmonellosis among yaks in several farms. We provided a comprehensive analysis of the molecular typing, WGS analysis, and the virulence of $S$. Derby isolated from yaks. This study reports the prevalence of $S$. Derby in yaks with diarrhea and in their drinking water. Although the clinical signs and contemporaneous occurrence of the outbreaks in all 13 farms suggested a diagnosis of Salmonella spp. infection, $S$. Derby was not isolated from yak feces taken from five farms (F1, F4, F5, F7, and F10). We speculated that the harsh and complex environment in the plateau area such as low temperature might have caused the yak diarrhea, which is a reminder that many more factors should be considered in future investigations.

Host adaptation is the ability of a serovar to circulate and persist in the population of a particular host species and may also be associated with distinct outcomes of infection. A previous study found that the STs of $S$. Derby corresponded to different hosts [57]. The most prevalent sequence type (ST) of $S$. Derby is ST40, which is considered to be well-adapted to swine and turkey $[18,58]$. A total of 2682 records of $S$. Derby ST40 from more than 20 countries have been uploaded to the EnteroBase Salmonella database (accessed 1 April 2021), indicating the international spread of ST40. In this study, there was no significant difference between the isolates at the genetic level. The 32 strains of $S$. Derby were all ST40, while ST40 was rarely found in yaks. In a previous study, the SPI- 23 was only present in the swine-adapted lineages ST40 and ST39 [59,60]. Hayward et al. hypothesized that the host adaption of $S$. Derby might be distinguished by the presence and absence of SPI- 23 . In our study, we found that all 32 strains of $S$. Derby lacked SPI-23, which may provide further evidence for the hypothesis. Further investigation and characterization are required to verify the relationship between SPIs and S. Derby in host adaption.

All $S$. Derby isolates from both fecal and drinking water samples from 13 farms were clonally related based on SNP alignment (Figure 2). The SNP differences between water samples (SD-1-11, SD-5-15, SD-13-18, and SD-13-19) were between four and five; the SPN differences between isolates from some farms were within five. The 13 farms surveyed were geographically distant from each other (Figure 1); the herders of each farm also seldom communicated and gathered. There was no transportation of yaks between each of the farms, and the yaks from each farm fed on the grass from their own grazing area throughout the year. Therefore, the possibility of salmonella transmission due to yak transfer is low. Some of the fecal samples, especially diarrhea fecal samples that were not easy to pick up, would flow into the river, which ran through local farms. The yaks in the surveyed farms used the river as a source of drinking water. Therefore, this may have infected the yaks as salmonella could survive in the water environment for a long time. The results may thus establish the hypothesis that there might be a clonal transmission between farms and a cross-contamination of salmonella.

We investigated the effect of infection caused by $S$. Derby in KM mice to better understand the progression of salmonellosis induced by $S$. Derby. In the pathogenicity study, the $\mathrm{LD}_{50}$ value of the original yak $S$. Derby was $4.57 \times 10^{7} \mathrm{CFU}$, which indicated less pathogenicity than other Salmonella serovars and was consistent with previous studies [61-63]. Shedding of the $S$. Derby strain used was high at three days post-injection (DPI), and the maximum amount of salmonella excreted was observed at nine DPI; the $S$. Derby was detected positively at $24 \mathrm{DPI}$, at $2.15 \times 10^{6} \mathrm{CFU} / \mathrm{g}$ of feces in mice, which showed that the bacterial shedding of feces was prolonged. Collectively, we established a KM mouse model to study infection by the $S$. Derby isolate and conducted its $\mathrm{LD}_{50}$, duration, and bacterial loads in organs. The findings of the present study demonstrated 
that $S$. Derby isolated from yak feces and their drinking water could successfully infect $\mathrm{KM}$ mice, leading to obvious clinical symptoms at high infectious doses. S. Derby also induced pathological damages to different organs in KM mice. The mice model for $S$. Derby infection established in this study provides an experimental animal model for the study of $S$. Derby infection and pathogenesis, and lays a foundation for further research. In the future, we intend to return to conduct relevant research on the yaks in order to provide more data for local salmonella control.

\section{Conclusions}

Salmonella spp. is a significant pathogen in food-producing animals, which are the primary source of salmonellosis [64]. Once introduced into the population, this can have widespread and serious consequences [65]. Thirteen geographically distinct farms were investigated for an outbreak of salmonellosis in yaks, with $32 \mathrm{~S}$. Derby strains being isolated from 162 samples of feces and drinking water. These isolates were all ST40, which was considered to be well-adapted to swine and turkey, and all of them lacked the SPI-23. Otherwise, the phylogenetic tree and WGS analyses showed that these isolated strains were clonally related, indicating the clonal spread of the strains. Infection of yaks with $S$. Derby could facilitate the dissemination of salmonella into the food chain and its eventual transmission to humans. Furthermore, our study proved that $S$. Derby could induce pathological damages in KM mice. With the intrusive and damaging effects in KM mice, these results thus lay the basis for further studies on the virulence of $S$. Derby in yaks and humans alike. The present study also demonstrated the prevalence of $S$. Derby in yaks with diarrhea and in their drinking water, as well as its pathogenicity in mice. This study adds to our understanding of the $S$. Derby serovar isolated from yaks. Seeing that $S$. Derby has become one of the most common Salmonella serovars, this situation gives rise to further risk from the potential spread of food-borne diseases.

Supplementary Materials: The following are available online at https://www.mdpi.com/article/ 10.3390/ani11082397/s1, Table S1: Prevalence of Salmonella in yaks with diarrhea in Hongyuan, Tibetan Qiang Autonomous Prefecture of Ngawa, China, Table S2: Overview of complete genome sequence information of 32 salmonella strains.

Author Contributions: Conceptualization, X.F., L.F. and L.K.; methodology, L.F.; software, X.F.; validation, X.F. and L.F.; formal analysis, L.F.; investigation, X.F., L.F., P.C., Y.Z., and H.L. (Hao Li); resources, X.Z., H.L. (Huade Li) and C.L.; data curation, X.F. and L.F.; writing-original draft preparation, L.F.; writing—review and editing, X.F.; visualization, W.Y. and L.Z.; supervision, H.W. and X.Y.; project administration, X.Y.; funding acquisition, X.Z. All authors have read and agreed to the published version of the manuscript.

Funding: This study was funded by the National Key Research Program of China (2017YFD0502200), and the Project for Science and Technology Support Program of Sichuan Province (2016NZ0005).

Institutional Review Board Statement: The mice were treated based on the guidelines of the Committee for the Control and Supervision of Animal Experiments, and all protocols were approved by the Institutional Animal Ethics Committee (License: SYXK (Chuan) 2013-185) of Sichuan University.

Data Availability Statement: The Bioproject numbers of the 32 strains of Salmonella in GenBank were PRJNA610941 and PRJNA640879.

Acknowledgments: The authors would like to thank all the reviewers who participated in the review and MJEditor (www.mjeditor.com, accessed on 19 April 2021) for its linguistic assistance during the preparation of this manuscript.

Conflicts of Interest: The authors declare no conflict of interest. The funders had no role in the design of the study; in the collection, analyses, or interpretation of data; in the writing of the manuscript, or in the decision to publish the result. 


\section{References}

1. Hoelzer, K.; Cummings, K.J.; Wright, E.M.; Rodriguez-Rivera, L.D.; Roof, S.E.; Switt, A.M.; Dumas, N.; Root, T.; Schoonmaker-Bopp, D.J.; Grohn, Y.T.; et al. Salmonella Cerro isolated over the past twenty years from various sources in the US represent a single predominant pulsed-field gel electrophoresis type. Vet. Microbiol. 2011, 150, 389-393. [CrossRef] [PubMed]

2. Navarro-Gonzalez, N.; Mentaberre, G.; Porrero, C.M.; Serrano, E.; Mateos, A.; Lopez-Martin, J.M.; Lavin, S.; Dominguez, L. Effect of cattle on Salmonella carriage, diversity and antimicrobial resistance in free-ranging wild boar (Sus scrofa) in Northeastern Spain. PLoS ONE 2012, 7, e51614. [CrossRef] [PubMed]

3. Nova, M.V.; Durimel, K.; La, K.; Felten, A.; Bessières, P.; Mistou, M.Y.; Mariadassou, M.; Radomski, N. Genetic and metabolic signatures of Salmonella enterica subsp. enterica associated with animal sources at the pangenomic scale. BMC Genom. 2019, 20, 814.

4. Jin, H.K.; Kim, H.J.; Jung, S.J.; Mizan, M.R.; Si, H.P.; Ha, S. Characterization of Salmonella spp.-specific bacteriophages and their biocontrol application in chicken breast meat. J. Food Sci. 2020, 85, 526-534.

5. Albino, L.A.; Rostagno, M.H.; Húngaro, H.M.; Mendonça, R.C. Isolation, characterization, and application of bacteriophages for Salmonella spp. biocontrol in pigs. Foodborne Pathog. Dis. 2014, 11, 602-609. [CrossRef] [PubMed]

6. Hassan, F.A.; Ibrahim, M.; Arafa, S.A. Effect of dietary pomegranate by-product extract supplementation on growth performance, digestibility, and antioxidant status of growing rabbit. Trop. Anim. Health Prod. 2020, 52, 1893-1901. [CrossRef]

7. Cetin, E.; Temelli, S.; Eyigor, A. Nontyphoid Salmonella prevalence, serovar distribution and antimicrobial resistance in slaughter sheep. Food Sci. Anim. Resour. 2019, 40, 21-33. [CrossRef]

8. Obaidat, M.M. Prevalence and antimicrobial resistance of Listeria monocytogenes, Salmonella enterica and Escherichia coli O157:H7 in imported beef cattle in Jordan. Comp. Immunol. Microbiol. Infect. Dis. 2020, 70, 101447. [CrossRef]

9. Xia, S.; Hendriksen, R.S.; Xie, Z.; Huang, L.; Zhang, J.; Guo, W.; Xu, B.; Ran, L.; Aarestrup, F.M. Molecular characterization and antimicrobial susceptibility of Salmonella isolates from infections in humans in Henan Province, China. J. Clin. Microbiol. 2009, 47, 401-409. [CrossRef]

10. Gu, D.; Wang, Z.; Tian, Y.; Kang, X.; Jiao, X. Prevalence of Salmonella isolates and their distribution based on whole-genome sequence in a chicken slaughterhouse in Jiangsu, China. Front. Vet. Sci. 2020, 7, 29. [CrossRef]

11. Xu, Z.; Song, Q.; Li, C.; Zhan, Y. Characterization of ciprofloxacin-resistant and ESBL-producing Salmonella enteric serotype Derby in Eastern China. BMC Microbiol. 2019, 19, 61. [CrossRef]

12. Ebuchi, S.; Ai, B.; Uryu, K.; Hiwaki, H. Two outbreaks caused by Salmonella Derby and S. Anatum at grilled-meat restaurants in Fukuoka city. Jpn. J. Infect. Dis. 2006, 59, 405-406.

13. Hendriksen, R.S.; Vieira, A.R.; Karlsmose, S.; Lo, F.; Jensen, A.B.; Wegener, H.C.; Aarestrup, F.M. Global monitoring of Salmonella serovar distribution from the World Health Organization Global Foodborne Infections Network Country Data Bank: Results of quality assured laboratories from 2001 to 2007. Foodborne Pathog. Dis. 2011, 8, 887-900. [CrossRef]

14. Jackson, B.R.; Griffin, P.M.; Cole, D.; Walsh, K.A.; Chai, S.J. Outbreak-associated Salmonella enterica serotypes and food Commodities, United States, 1998-2008. Emerg. Infect. Dis. 2013, 19, 1239-1244. [CrossRef]

15. Arnedo-Pena, A.; Sabater-Vidal, S.; Herrera-León, S.; Bellido-Blasco, J.B.; Silvestre-Silvestre, E.; Meseguer-Ferrer, N.; Yague-Munoz, A.; Gil-Fortuno, M.; Romeu-García, A.; Moreno-Munoz, R. An outbreak of monophasic and biphasic Salmonella Typhimurium, and Salmonella Derby associated with the consumption of dried pork sausage in Castellon (Spain). Enferm. Infecc. Y Microbiol. Clin. 2016, 34, 544-550. [CrossRef] [PubMed]

16. Sévellec, Y.; Felten, A.; Radomski, N.; Granier, S.A.; Hello, S.L.; Petrovska, L.; Mistou, M.Y.; Cadel-Six, S. Genetic diversity of Salmonella derby from the poultry sector in europe. Pathogens 2019, 8, 46. [CrossRef]

17. Schmidt, J.W.; Brichta-Harhay, D.M.; Kalchayanand, N.; Bosilevac, J.M.; Shackelford, S.D.; Wheeler, T.L.; Koohmaraie, M. Prevalence, enumeration, serotypes, and antimicrobial resistance phenotypes of Salmonella enterica isolates from carcasses at two large United States pork processing plants. Appl. Environ. Microbiol. 2012, 78, 2716. [CrossRef]

18. Cai, Y.; Tao, J.; Jiao, Y.; Fei, X.; Zhou, L.; Wang, Y.; Zheng, H.; Pan, Z.; Jiao, X. Phenotypic characteristics and genotypic correlation between Salmonella isolates from a slaughterhouse and retail markets in Yangzhou, China. Int. J. Food Microbiol. 2016, $222,56-64$. [CrossRef]

19. Xu, C.; Ren, X.; Feng, Z.; Fu, Y.; Hong, Y.; Shen, Z.; Zhang, L.; Liao, M.; Xu, X.; Zhang, J. Phenotypic characteristics and genetic Diversity of Salmonella enterica serotype derby isolated from human patients and foods of animal origin. Foodborne Pathog. Dis. 2017, 14, 593-599. [CrossRef]

20. Authority, E. The European Union summary report on trends and sources of zoonoses, zoonotic agents and food-borne outbreaks in 2016. EFSA J. 2017, 15, e05077.

21. Gong, X.; Liu, L.; Zheng, F.; Chen, Q.; Li, Z.; Cao, X.; Yin, H.; Zhou, J.; Cai, X. Molecular investigation of bovine viral diarrhea virus infection in yaks (Bos gruniens) from Qinghai, China. Virol. J. 2014, 11, 1-7. [CrossRef]

22. Cui, P.; Feng, L.; Zhang, L.; He, J.; Yang, X. Antimicrobial resistance, virulence genes, and biofilm formation capacity among Enterococcus species from Yaks in Aba Tibetan autonomous prefecture, China. Front. Microbiol. 2020, 11, 1250. [CrossRef] [PubMed]

23. Li, K.; Zhang, L.; Zhang, H.; Lei, Z.; Luo, H.; Mehmood, K.; Shahzad, M.; Lan, Y.; Wang, M.; Li, J. Epidemiological investigation and risk factors of Echinococcus granulosus in yaks (Bos grunniens), Tibetan pigs and Tibetans on Qinghai Tibetan plateau. Acta Trop. 2017, 173, 147-152. [CrossRef] [PubMed] 
24. Feng, L.; An, T.; Cui, P.; Zhao, X.; Li, H.; Yang, X. Isolation, identification, and pathogenicity experiment of Salmonella enterica serovar derby from yaks (Poephagus grunniens) with diarrhea in Tibetan Qiang Autonomous Prefecture of Ngawa, China. J. Sichuan Univ. 2020, 57, 1022-1030.

25. Tsai, H.L.; Hsu, B.M.; Hsu, T.K.; Huang, K.H.; Shih, F.C.; Chen, J.S.; Wang, H.J.; Kao, P.M.; Su, H.C. Evaluation of immunomagnetic separation for the improvement of Salmonella detection in aquatic environment. Environ. Earth Sci. 2014, 73, 7909-7914. [CrossRef]

26. Popoff, M.Y.; Minor, L.L. Antigenic Formulas of the Salmonella Serovars; 8th Revision; WHO Collaborating Center for Reference and Research on Salmonella, Institute Pasteur: Paris, France, 2001.

27. Basic Local Alignment Search Tool. Available online: https://blast.ncbi.nlm.nih.gov/Blast.cgi (accessed on 23 April 2021).

28. Queipo-Ortuño, M.I.; De Dios Colmenero, J.; Macias, M.; Bravo, M.J.; Morata, P. Preparation of bacterial DNA template by boiling and effect of immunoglobulin $\mathrm{G}$ as an inhibitor in real-time PCR for serum samples from patients with brucellosis. Clin. Vaccine Immunol. 2008, 15, 293. [CrossRef]

29. Wang, Q.; Hu, C.; Ke, F.; Huang, S.; Li, Q. Characterization of a bacterial biocontrol strain 1404 and its efficacy in controlling postharvest citrus anthracnose. Acta Microbiol. Sin. 2010, 50, 1208-1217.

30. Overbeek, R.; Olson, R.; Pusch, G.D.; Olsen, G.J.; Stevens, R. The SEED and the Rapid Annotation of microbial genomes using Subsystems Technology (RAST). Nucleic Acids Res. 2013, 42, D206-D214. [CrossRef]

31. Kidgell, C.; Reichard, U.; Wain, J.; Linz, B.; Achtman, M. Salmonella typhi, the causative agent of typhoid fever, is approximately 50,000 years old. Infect. Genet. Evol. 2002, 2, 39-45. [CrossRef]

32. EnteroBase. Available online: http://enterobase.warwick.ac.uk/species/index/senterica (accessed on 23 April 2021).

33. Nabil-Fareed, A.; Zhou, Z.; Sergeant, M.J.; Mark, A.; Josep, C. A genomic overview of the population structure of Salmonella. PLoS Genet. 2018, 14, e1007261.

34. Jolley, K.A.; Bliss, C.M.; Bennett, J.S.; Bratcher, H.B.; Brehony, C.; Colles, F.M.; Wimalarathna, H.; Harrison, O.B.; Sheppard, S.K.; Cody, A.J.; et al. Ribosomal multilocus sequence typing: Universal characterization of bacteria from domain to strain. Microbiology 2012, 158, 1005-1015. [CrossRef]

35. Kaas, R.S.; Leekitcharoenphon, P.; Aarestrup, F.M.; Lund, O. Solving the problem of comparing whole bacterial genomes across different sequencing platforms. PLoS ONE 2014, 9, e104984. [CrossRef]

36. Tamura, K.; Nei, M. Estimation of the number of nucleotide substitutions in the control region of mitochondrial DNA in humans and chimpanzees. Mol. Biol. Evol. 1993, 10, 512-526. [PubMed]

37. Kumar, S.; Stecher, G.; Tamura, K. MEGA7: Molecular evolutionary genetics analysis version 7.0 for bigger datasets. Mol. Biol. Evol. 2016, 33, 1870-1874. [CrossRef]

38. Interactive Tree of Life (iTOL) v6. Available online: https://itol.embl.de/itol.cgi (accessed on 20 April 2021).

39. Dallman, T.J.; Lisa, B.; Ashton, P.M.; Cowley, L.A.; Perry, N.T.; Goutam, A.; Liljana, P.; Ellis, R.J.; Richard, E.; Anthony, U. Whole-genome sequencing for national surveillance of shiga toxin-producing Escherichia coli O157. Clin. Infect. Dis. 2015, 61, 305-312. [CrossRef] [PubMed]

40. Kanagarajah, S.; Waldram, A.; Dolan, G.; Jenkins, C.; Ashton, P.M.; Martin, A.; Davies, R.; Frost, A.; Dallman, T.J.; Pinna, E. Whole genome sequencing reveals an outbreak of Salmonella Enteritidis associated with reptile feeder mice in the United Kingdom, 2012-2015. Food Microbiol. 2017, 71, 32-38. [CrossRef] [PubMed]

41. Valeria, B.; Kaas, R.S.; Etienne, R.; Roberts, M.C.; Stefan, S.; Vincent, C.; Alain, P.; Allesoe, R.L.; Rita, R.A.; Ferrer, F.A. ResFinder 4.0 for predictions of phenotypes from genotypes. J. Antimicrob. Chemother. 2020, 75, 3491-3500.

42. Roer, L.; Hendriksen, R.S.; Leekitcharoenphon, P.; Lukjancenko, O.; Kaas, R.S.; Hasman, H.; Aarestrup, F.M. Is the evolution of Salmonella enterica subsp. enterica linked to restriction-modification systems? Msystems 2016, 1, e00009-e00016. [CrossRef]

43. Liu, B.; Zheng, D.; Jin, Q.; Chen, L.; Yang, J. VFDB 2019: A comparative pathogenomic platform with an interactive web interface. Nucleic Acids Res. 2018, 47, 687-692. [CrossRef]

44. Pizzi, M. Sampling variation of the fifty percent end-point, determined by the Reed-Muench (Behrens) method. Hum. Biol. 1950, 22, 151-190.

45. Cevallos-Almeida, M.; Houdayer, C.; Rose, V.; Bailly, Y.; Paboeuf, F.; Fablet, C.; Denis, M.; Kerouanton, A. Colonization of pigs experimentally infected with a monophasic variant of Salmonella Typhimurium. Foodborne Pathog. Dis. 2018, 15, 576-582. [CrossRef] [PubMed]

46. Royston, P. A Remark on algorithm as 181: The W test for normality. J. R. Stat. Soc. Ser. C 1995, 44, 547-551.

47. Cimolai, N.; Taylor, G.P.; Mah, D.; Morrison, B.J. Definition and application of a histopathological scoring scheme for an animal model of acute mycoplasma pneumoniae pulmonary infection. Microbiol. Immunol. 1992, 36, 465-478. [CrossRef]

48. Oueslati, W.; Rjeibi, M.R.; Mhadhbi, M.; Jbeli, M.; Zrelli, S.; Ettriqui, A. Prevalence, virulence and antibiotic susceptibility of Salmonella spp. strains, isolated from beef in Greater Tunis (Tunisia). Meat Sci. 2016, 119, 154-159. [CrossRef]

49. Mustefa, B.A.; Gebremedhin, E.Z. Carriage and antimicrobial resistance of non-typhoidal Salmonella in cattle slaughtered in Ambo municipality abattoir, West Shewa zone, Oromia, Ethiopia-A point prevalence survey. Ethiop. Vet. J. 2018, 22, 94. [CrossRef]

50. Sychanh, T.; Chaunchom, S.; Pulsrikarn, C.; Pornreongwong, S.; Boonmar, S. Salmonella prevalence in slaughtered buffaloes and cattle in Champasak province, Lao People's Democratic Republic. Agric. Nat. Resour. 2013, 47, 561-570.

51. Fedorka-Cray, P.J.; Dargatz, D.A.; Thomas, L.A.; Gray, J.T. Survey of Salmonella serotypes in feedlot cattle. J. Food Prot. 1998, 61, 525-530. [CrossRef] 
52. Guizelini, C.C.; Tutija, J.F.; Morais, D.R.; Bacha, F.B.; Ramos, C.A.N.; Leal, C.R.B.; Zaquetti, M.E.; Lemos, R.A.A. Outbreak investigation of septicemic salmonellosis in calves. J. Infect. Dev. Ctries. 2020, 14, 104-108. [CrossRef]

53. Salaheen, S.; Sonnier, J.; Kim, S.W.; Haley, B.J.; Van Kessel, J.A.S. Interaction of Salmonella enterica with bovine epithelial cells demonstrates serovar-specific association and invasion patterns. Foodborne Pathog. Dis. 2020, 17, 608-610. [CrossRef]

54. Hoelzer, K.; Switt, A.I.M.; Wiedmann, M. Animal contact as a source of human non-typhoidal salmonellosis. Vet. Res. 2011, 42, 1-28. [CrossRef]

55. Guo, X.; Long, R.; Kreuzer, M.; Ding, L.; Shang, Z.; Zhang, Y.; Yang, Y.; Cui, G. Importance of functional ingredients in yak milk-derived food on health of Tibetan nomads living under high-altitude stress: A review. Crit. Rev. Food Sci. Nutr. 2014, 54, 292-302. [CrossRef]

56. Hayward, M.R.; Petrovska, L.; Jansen, V.; Woodward, M.J. Population structure and associated phenotypes of Salmonella enterica serovars Derby and Mbandaka overlap with host range. BMC Microbiol. 2016, 16, 1-9. [CrossRef]

57. EFSA. The European Union One Health 2018 Zoonoses Report. EFSA J. 2019, 17, e05926.

58. Yann, S.; Marie-Léone, V.; Granier, S.A.; Renaud, L.; Carole, F.; Le, H.S.; Michel-Yves, M.; Sabrina, C.S. Polyphyletic Nature of Salmonella enterica Serotype Derby and Lineage-Specific Host-Association Revealed by Genome-Wide Analysis. Front. Microbiol. 2018, 9, 891.

59. Hayward, M.R.; Jansen, V.A.; Woodward, M.J. Comparative genomics of Salmonella enterica serovars Derby and Mbandaka, two prevalent serovars associated with different livestock species in the UK. BMC Genom. 2013, 14, 365. [CrossRef] [PubMed]

60. Hayward, M.R.; Abuoun, M.; Ragione, R.; Tchórzewska, M.; Woodward, M.J. SPI-23 of S. Derby: Role in adherence and invasion of porcine tissues. PLOS ONE 2014, 9, e107857.

61. Olsen, J.E.; Brown, D.J.; Thomsen, L.E.; Platt, D.J.; Chadfield, M.S. Differences in the carriage and the ability to utilize the serotype associated virulence plasmid in strains of Salmonella enterica serotype Typhimurium investigated by use of a self-transferable virulence plasmid, pOG669. Microb. Pathog. 2004, 36, 337-347. [CrossRef]

62. Swearingen, M.C.; Porwollik, S.; Desai, P.T.; Mcclelland, M.; Ahmer, B. Virulence of 32 Salmonella strains in mice. PLoS ONE 2012, 7, e36043. [CrossRef]

63. Tennant, S.M.; Schmidlein, P.; Simon, R.; Pasetti, M.F.; Levine, M.M. Refined live attenuated Salmonella enterica Serovar typhimurium and enteritidis vaccines mediate homologous and heterologous serogroup protection in mice. Infect. Immun. 2015, 83, 4504-4512. [CrossRef] [PubMed]

64. Plym, F.L.; Wierup, M. Salmonella contamination: A significant challenge to the global marketing of animal food products. Rev. Sci. Tech. 2006, 25, 541-554.

65. Lamas, A.; Miranda, J.M.; Regal, P.; Vázquez, B.; Franco, C.M.; Cepeda, A. A comprehensive review of non-enterica subspecies of Salmonella enterica. Microbiol. Res. 2018, 206, 60-73. [CrossRef] [PubMed] 